\title{
Adaptive Overtaking Control and Effective Power Generation (AOC-EPG) System
}

\author{
Aravindan Anbarasu. \\ Kumaraguru College of Technology, INDIA
}

\begin{abstract}
The innovative idea Adaptive Overtaking Control and Effective Power Generation (AOC-EPG) system comes into action to prevent accidents that are caused due to lack of concentration and helps in power generation.(AOC-EPG) prevents collision of cars during overtaking. It consists of ultrasonic transducers, for effective measurement of distance between two vehicles, by the usage of microcontroller. In a series path of three cars $A, B \& C$, If the car $(B)$ wants to overtake and if there is a possibility, that is calculated using microcontroller based ultrasonic system which locates the vehicle coming in the opposite direction, from its path itself. And then an information is communicated to the car $(A)$ in front by using RF transceiver of it, that it is going to overtake and simultaneously the length of the $\operatorname{car}(B)$ is communicated to the $\operatorname{car}(C)$ behind it by using same RF transceiver, so that the car $(C)$ maintains the displaced length until the overtaking is done. If there is no possibility of overtaking and still it is overruled (AOC-EPG) system prevents further acceleration towards the other side, thus the driver has no choice and hence he has to come back to his former place.

$(A O C-E P G)$ also supports an important feature called the regenerative power from manual/automatic transmission, which ensures that the kinetic energy wasted during the gear shifts can be effectively used in generating power, thus powering the needs of the car electronic systems and if the power generated is more, then it is stored in a separate battery, such that it can be used to power devices in home - a concept called Vehicle to Home Power Transfer System.
\end{abstract}

Keywords: Renesas R8C Controller, Ultrasonic Sensors, RF Transceiver, alternator, flywheel.

\section{Introduction}

A quarter of our lives is spend in cars and other travelling mediums, but at the same time a quarter of population lose their life due to potential accidents and the main reason is the lack of concentration and overconfidence while driving. Blindfolded overtaking is one of the main reasons considered for catastrophic accidents. In today's world in a common man's view "Rules are made to be Broken". So Rapid development is taking place in innovating a device, that is intelligent enough to prevent accidents and making sure that rules are followed by people for a safe and pleasure driving. Adaptive Overtaking Control and Effective Power Generation (AOC-EPG) System is one such device, which could keep accidents at bay; simultaneously seeing to that both car and home is powered by the effective conversion of energy.

\section{Main Concept}

The overview of the entire system is shown in fig 1, with microcontroller as the heart. It consists of 2 segments each designed for a specific purpose. They are

1. Ultrasonic-based distance measurement

a. Output-display unit

b. Communication-RF Transceiver

2. Regenerative power generation

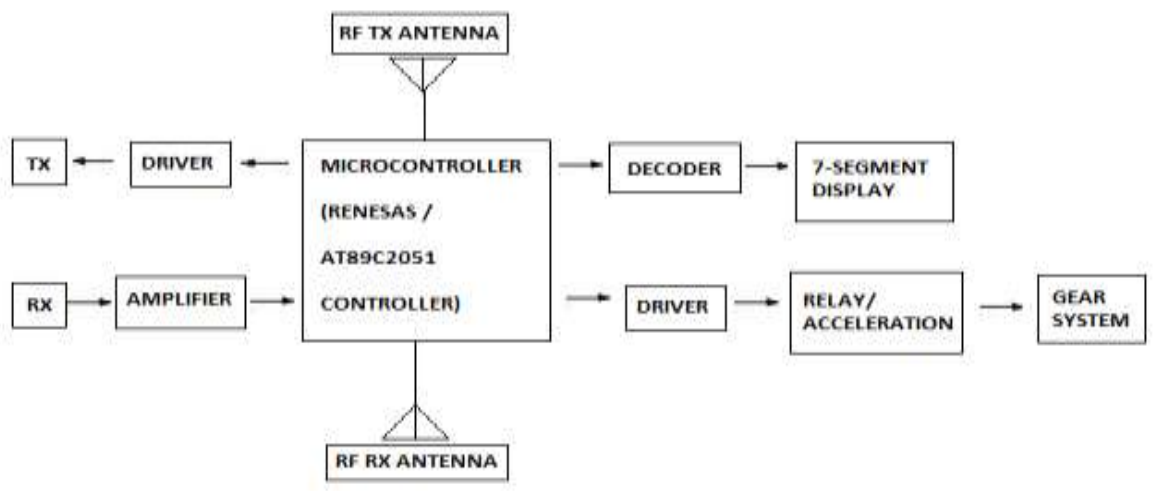

Fig.1: General Block Diagram 


\section{Microcontroller-Based Distance Measurement}

The distance is calculated using ultrasonic transducers. The required components are AT89C2051 microcontroller (Renesas R8C Controller can be used for voice or audio message, i.e., instead of having seven segment display, speakers can be used to hear the voice message.), two $40 \mathrm{KHz}$ ultrasonic transducers with few discreet components. They measure the amount of time taken for a pulse of sound to travel to a particular target and return as the reflected echo shown in Fig.2.

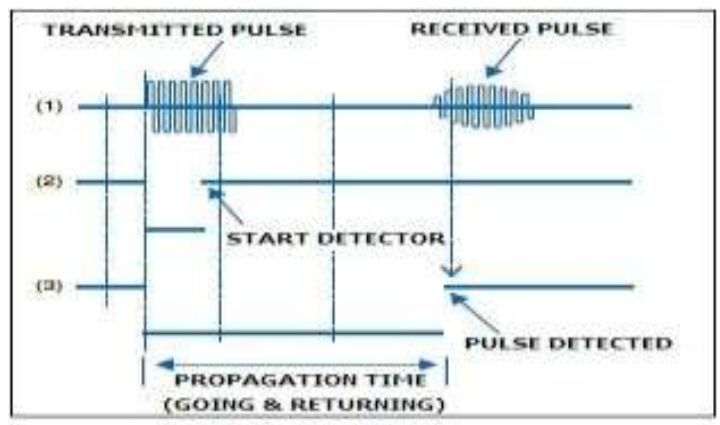

Fig.2: Ultrasonic pulse, Echo Signal and Tim Measurement

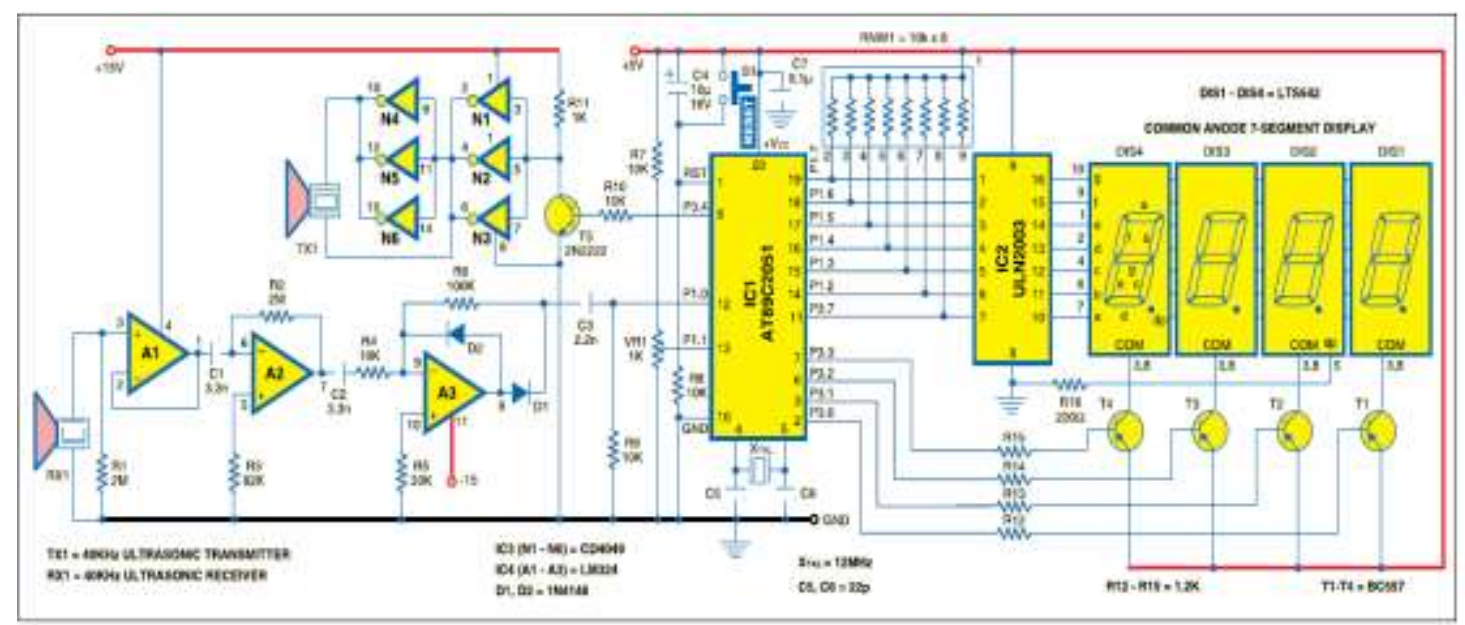

Fig.4: Circuit Diagram

From the reflected wave, microcontroller calculates the distance based on the speed of sound and displays it in a 7 segment display. At the receiving end, level control is used in order to eliminate the side lobe as main lobe would only give the correct distance as shown in the figure Fig.3

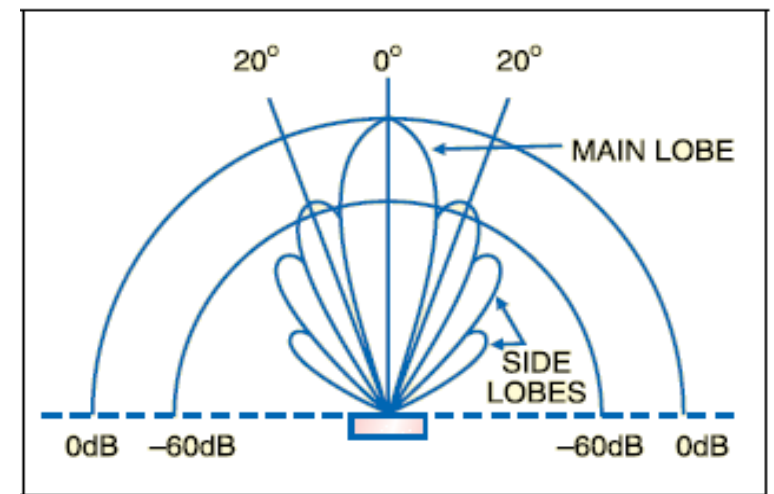

Fig.3: 2D Beam Pattern of Ultrasonic Signal Showing Main Lobe and Side Lobe Energy Levels.

\section{Transmitter}

The $40 \mathrm{kHz}$ pulse bursts from the microcontroller are amplified by transistorT5. Inverting buffer CD4049 drives the ultrasonic sensor used as the transmitter. Outputs of both sets of parallel inverters are applied 
as a push pull drive to the ultrasonic transmitter. The positive going pulse is applied to one of the terminals of the ultrasonic sensor and the same pulse after 180-degree phase shift is applied to another terminal.

\section{Receiver}

The echo signal received by the receiver sensor after reflection is very weak. It is amplified by quad operational amplifier LM324. When port-3 pin P3.6 goes high, we know that the echo signal has arrived the timer is read and the 16-bit number is divided by twice the velocity of sound and then converted into a decimal format and displaced as a 4-digit number, using seven segment displays as shown in Fig .4

\section{Adaptive Overtaking}

Fig.5.1, represents that car B preparing to overtake by sliding the ultrasonic transceiver to the opposite lane.

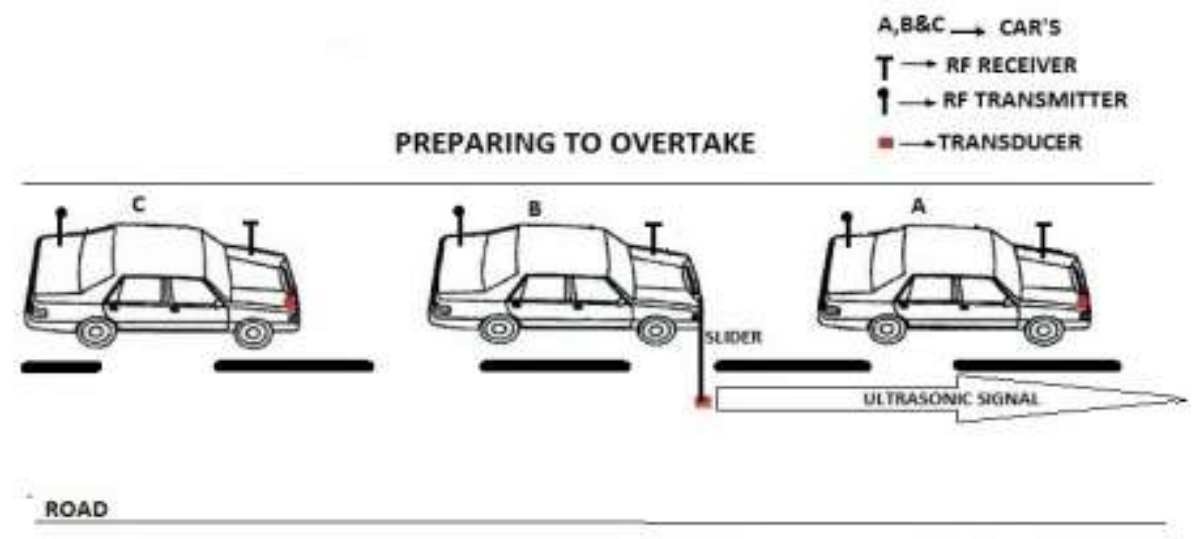

Fig.5.1: Overtaking Decision

Fig $5.2 \& 5.3$ represents the action of the car while overtaking process when there is any incoming car (car in opposite direction) and in the absence of an incoming car respectively.

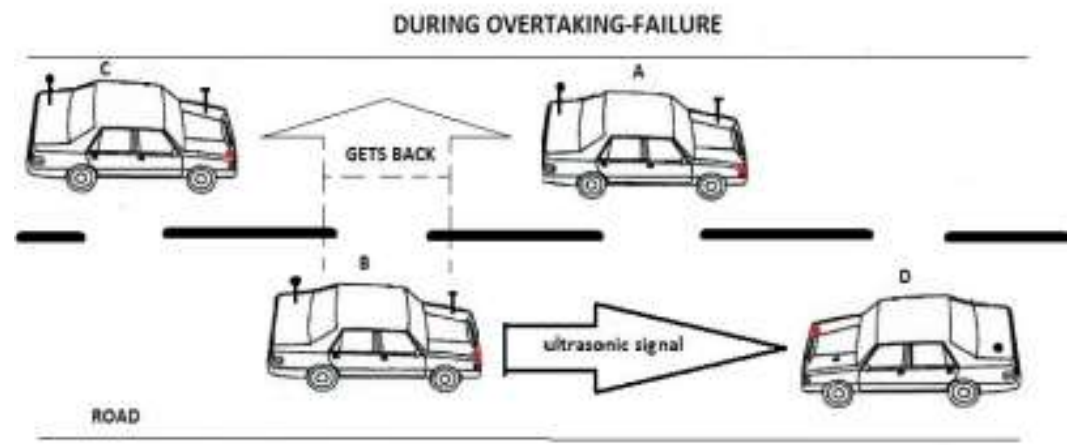

Fig.5.2: Action in Progress

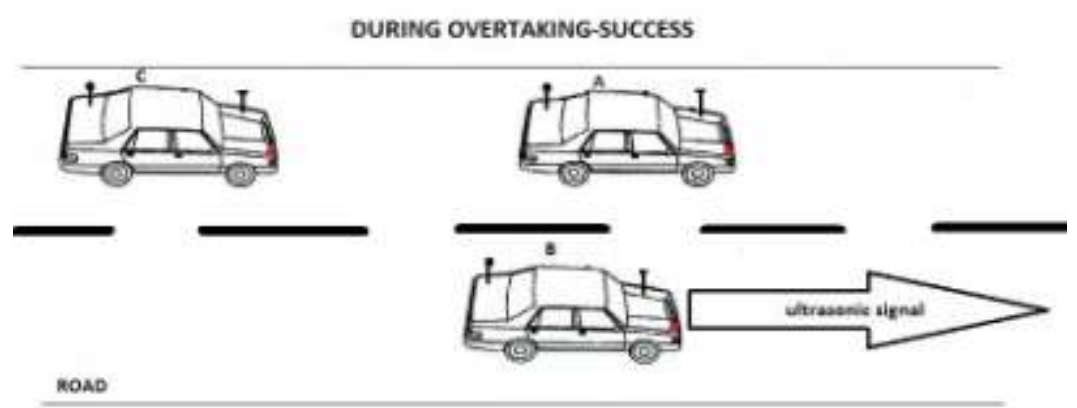

Fig.5.3: Action in Progress 
If the Adaptive Overtaking Control (AOC) Unit is not present in the cars then overtaking becomes a risky task for all the drivers, for example if car B is in the process of overtaking its place is taken by the car C, so in case of any interruption the car B has no place to come back to its former lane, leading to accident. Thus the Adaptive Overtaking Control makes the overtaking process a comfortable one for all the drivers and thereby reducing the car accidents.

\section{Power Generation System}

Power can be effectively generated from gear shift during manual/automatic transmission, thus opening a door for effective conversion and utilization of energy.

\section{A. Basic Principle And Necessacity}

It's a simple electromechanical device used to convert rotational energy into electrical energy. When a vehicle is running on a high gear, and suddenly for some reason it shifts the gear down to come to a lower speed, in that instance the kinetic energy possessed by the vehicle when it was in a high gear is lost when it shifts down to a lower gear. So here this device is implemented to use the kinetic energy lost between the gear shifts and convert it to equivalent electrical energy.

\section{B. Operation}

It consists of a flywheel connected with an alternator. A microcontroller is used to attach this setup to a higher gear, when the speed of vehicle exceeds $60 \mathrm{kmph}$ and it gets detached when the speed is less than $60 \mathrm{kmph}$. When it is attached, the flywheel rotates with speed of that gear and when the car shifts to a lower gear, for example $30 \mathrm{kmph}$, the setup is detached and continues to rotate at a speed when the car was in a higher gear for some time. As the flywheel rotates the alternator produces AC, which is converted to DC by a rectifier This DC is used to charge two sets of battery, one for the car and one for the home purpose. If the car battery has sufficient charge and does not need charging from this device, then we can switch it to a mode where it charges only the battery for the home usage, where a concept called Vehicle to Home power transfer system is implemented as shown in Fig.6,

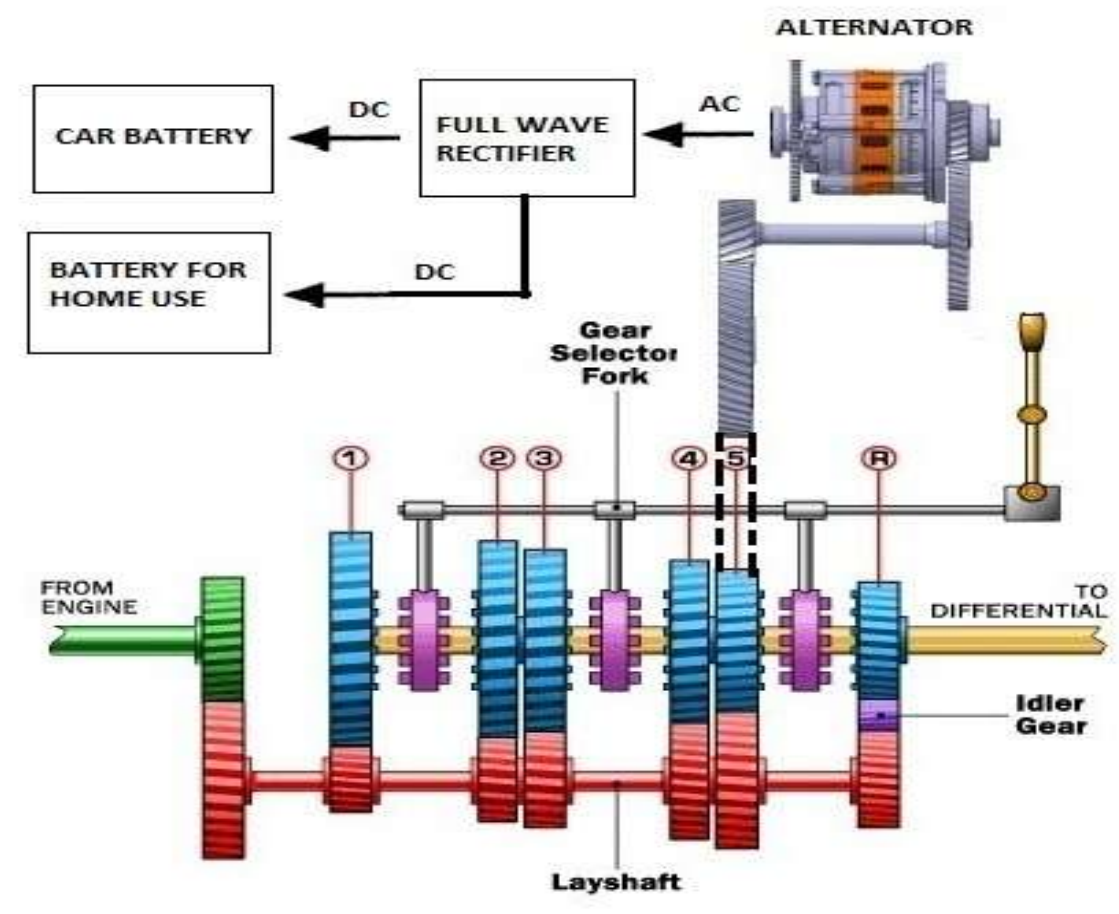

Fig. 6: EPG System 
VI. Adaptive Overtaking Control And Effective Power Generation System The functional Block diagram of the AOC-EPG is given below in Fig.7,

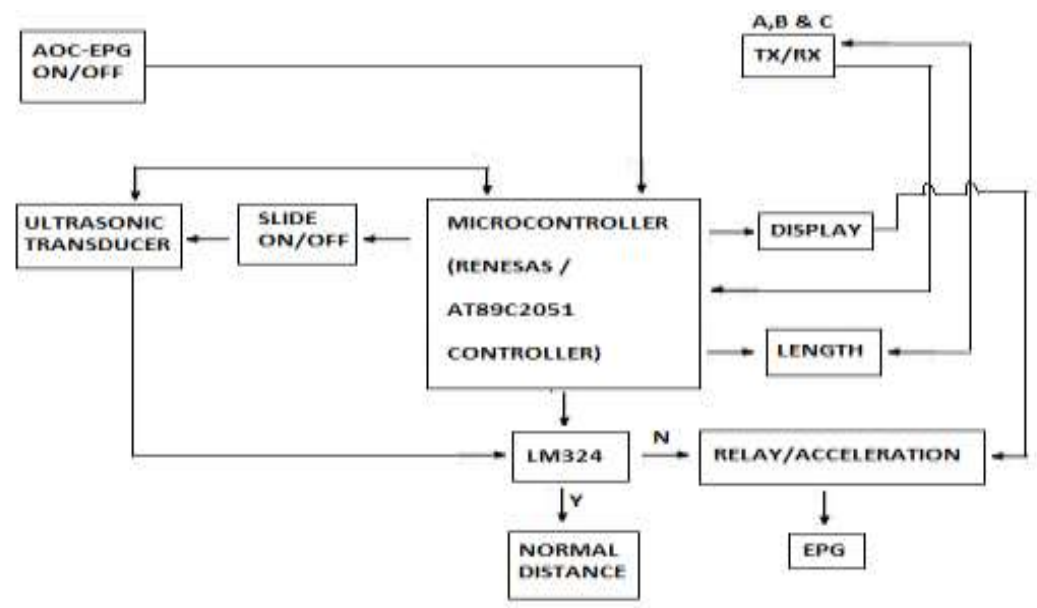

Fig.7: AOC-EPG System.

This system is present in each car A, B, C. The function of this system in each of the three cars is explained. When a car (B) wants to safely overtake, the AOC-EPG button is pressed by the driver. The moment it is pressed a slider with ultrasonic transducer slides towards the opposite lane to detect any incoming vehicle with a specific distance, after that it slides back. If a car is detected then there is no chance to change to the opposite lane but still if it is overruled then the microcontroller of car (B) sends signal to relay/acceleration unit (to decelerate) and EPG system.

If no incoming vehicle is detected, then the car comes to opposite lane. After that ultrasonic transducer is automatically enabled, for checking the possibility of overtaking by calculating the distance between the car and incoming vehicle (also the receiver antenna of that particular car will be turned off). While the car (B) is in the process of overtaking, its physical length is communicated to car(C) behind it using RF transceiver. The $\operatorname{car}(\mathrm{C})$ receives the signal and the microcontroller of it computes the critical distance using formula [physical length $+1 \mathrm{~m}$ ]. And it maintains a critical distance for the car (B) to again get back to its former lane in the event of failure of overtaking.

On receiving the data, the microcontroller of $\operatorname{car}(\mathrm{C})$ enables the ultrasonic transducers to calculate the distance between $\operatorname{car}(\mathrm{C}) \&$ car $(\mathrm{A})$. This calculated distance and the critical distance is compared using a comparator LM324, and decision is taken. If the distance is matched then, no action is taken by the microcontroller, else the microcontroller signals to the relay/acceleration unit thus enabling EPG system.

\section{Conclusion}

Thus the Adaptive Overtaking Control and Effective Power Generation (AOC-EPG) System helps in saving the lives which are lost when car accidents happen due to carelessness and lack of concentration of the driver and also it helps to meet the power requirement of both the car and home to some extent and thus opening a window for meeting the power crisis in most of the developing countries.

\section{Reference}

[1]. "Mechanical Design of Machines Elements and Machines"-by Jack A Collins, Henry R.Busby, George H.Staab

[2]. "Automotive Transmissions Fundamentals, Selection Design and Application"-by Hatald Naunheimer, bernd bertsche, Joachim Ryborz, Wolfgang Novak

[3]. Ultrasound transducer materials"-by Don Berlincourt, O.E Mattiat, Yoshmitsu Kikuchi

[4]. "Flywheel Energy calculator"-baltana.org

[5]. "Understanding how alternators work"- www.howstuffworks.com

[6]. "Accuracy and Resolution of ultrasonic distance measurement with pulse echo method"-www.kurosawa.ip.titech.ac.jp/.../ast09sh2.pdf

[7]. "Ultrasonic sound echoes"-hyperphysics.phy aster.gsu.edu/.../usound.html

[8] ."Manual Transmission"-www.howstuffworks.com 